\title{
Autologous Bone Marrow Mononuclear Cells may be Explored as a Novel Potential Therapeutic Option for Autism
}

\section{Alok Sharma1, Nandini Gokulchandran ${ }^{1}$, Akshata Shetty ${ }^{2}$, Hemangi Sane ${ }^{3}$, Pooja Kulkarni ${ }^{3 *}$ and Prerna Badhe $^{1}$}

${ }^{1}$ Department of Medical Services and Clinical research, NeuroGen Brain and Spine Institute, Surana Sethia Hospital and Research centre, Mumbai, India ${ }^{2}$ Department of NeuroRehabilitation, NeuroGen Brain and Spine Institute, Surana Sethia Hospital and Research centre, Mumbai, India

${ }^{3}$ Department of Research and Development, NeuroGen Brain and Spine Institute, Surana Sethia Hospital and Research centre, Mumbai, India

\begin{abstract}
Cellular therapy has been viewed as a novel therapeutic modality for many neurological disorders. Autologous Bone Marrow Mononuclear Cells (BMMNCs) used in many studies, have a safe and ethical profile. These cells have been studied in great depth and have shown angiogenetic and immunomodulatory properties in addition to other neuroprotective effects. These peculiar mechanisms may serve to be beneficial in autism. Recently, hypoperfusion and immune alteration are identified as major underlying pathogenetic mechanisms in autism. We present a case of autism with comorbid mental retardation; treated with intrathecal administration of autologous BMMNCs. Results were documented objectively on Indian Scale for Assessment of Autism (ISAA) and Positron Emission Tomography Computed Tomography (PET CT) scan. On regular follow up assessment of the patient over 18 months, there was significant clinical improvement in social relationship, communication and behavior. On outcome measure, ISAA score improved from 111 (Moderate autism) to 73 (Mild Autism). PET CT scans comparison of pre and post therapy showed balancing effect on brain metabolism. This case provides a great insight into the clinical effects of autologous BMMNCs in autism. Though a case study, the improvements guide us to plan future studies to explore different options of cellular therapy in autism.
\end{abstract}

Keywords: Autologous; Bone marrow; Mononuclear cells; Autism; ISAA; PET CT scan

\section{Introduction}

Autism spectrum disorders are a group of neurodevelopmental conditions characterized by difficulty in verbal and nonverbal communication, impairment in social interaction and restricted repetitive obsessive behaviour or interests. The caregivers usually notice the symptoms around 12-18 months and definitive diagnosis is usually made around 24-36 months [1]. However, in some cases the diagnosis may be done in adulthood. Autism is believed to occur at a rate of about 8 cases per 10,000 children ( 0.08 percent) [2]. Current treatment options available for autism are behavioural therapy, occupational therapy, speech therapy, nutritional guidance and pharmacological treatment. However, these treatment options are directed towards symptomatic changes in the child. Here we describe a case of autism that was treated with conventional medical treatment and therapies yet he continued to have major behavior and communication deficits. Cellular therapy has recently been viewed as a promising therapeutic modality which aims at restoring neural connections in the brain [3]. In this case study, we have demonstrated the use of autologous BMMNC as a beneficial therapeutic approach for autism as they are easily obtainable, safe i.e. no risk of Graft-Versus-Host-Disease (GVHD) and non-teratogenic. They do not have any ethical or moral issues. The main aim of this therapy is to reduce the severity of autism and thereby improve the overall functioning of the child.

\section{Case Presentation}

\section{Case report}

We present a case of a 14 year old male with Autism. He was a 20 days preterm baby born through normal vaginal delivery. Normal milestones were achieved but there was a regression in speech at $3 \frac{1}{2}$ years and was diagnosed with autism with high levels of serotonin. Parents noticed hyperactivity and poor social interaction. Stereotypical motor mannerisms, minimal social reciprocity and poor eye contact were present along with self-stimulatory behaviour like jumping, hand flapping and self-touching at private parts. Speech and language skills were deficient. He could answer questions either by pointing or by making sounds and follow simple commands. He was oriented to person and place but disoriented in time, date and day. Psychomotor activity level was decreased. Reaction time and tempo were slow. The form, content and possession of thought were also absent. His attention span and concentration were average. Neurologically, muscle tone and power in trunk and limb muscles were normal. Functionally, he was totally independent in most of his Activities of Daily Living (ADLs) but required supervision. In spite of regular rehabilitation, no improvement was seen with respect to behavior, social interaction or speech. Intelligent Quotient (IQ) on The Malin's Intelligence Scale for Indian Children was found to be 44, which suggested Moderate Mental Retardation. On Indian Scale for Assessment of Autism (ISAA), the score was 111 which categorized him as moderate autism. Magnetic resonance Imaging (MRI) of the brain was normal. Electroencephalography (EEG) did not show any epileptogenic focus. The brain Positron Emission Tomography (PET) scan was performed using a GE Millenium Discovery VH hawkeye Hybrid Gamma camera scanner and the radioisotope used was 18-F FDG. The PET images showed less FDG uptake only in the temporal lobe, Wernicke's, parietal, occipital and basal ganglia on the right side while, anterior cingulate and calcarine region on the left side of the brain. The FDG uptake was more in other areas as compared to these areas.

*Corresponding author: Pooja Kulkarni, NeuroGen Brain and Spine Institute Surana Sethia Hospital and Research centre, Suman Nagar, Sion- Trombay Road, Chembur, Mumbai-400071, India, Tel. +9122-25281610/ +9122-25283706; E-mail: poojakul28@gmail.com

Received April 23, 2013; Accepted May 22, 2013; Published May 24, 2013

Citation: Sharma A, Gokulchandran N, Shetty A, Sane H, Kulkarni P, et al. (2013) Autologous Bone Marrow Mononuclear Cells may be Explored as a Novel Potential Therapeutic Option for Autism. J Clin Case Rep 3: 282. doi:10.4172/21657920.1000282

Copyright: ( 2013 Sharma A, et al. This is an open-access article distributed under the terms of the Creative Commons Attribution License, which permits unrestricted use, distribution, and reproduction in any medium, provided the original author and source are credited. 


\section{Method}

Intrathecal administration of autologous bone marrow derived mononuclear cell (BMMNC) was used as a treatment method. Our protocol was based on the inclusion criterion as per the World Medical Associations Helsinki declaration [4]. It has been reviewed and approved by the Institutional committee for Stem cell Research and Therapy (IC-SCRT). The patient's parents were informed about the procedure and a duly filled informed consent form was obtained. Pre-procedure routine blood tests, urinalysis and chest $\mathrm{x}$-ray were done to rule out active infection and assess anaesthesia fitness. $300 \mathrm{mcg}$ of G-CSF injections were administrated 48 hours and 24 hours before bone marrow derived mononuclear cell transplantation, to stimulate CD34+ cells and increase their survival and multiplication. Bone marrow $(100 \mathrm{ml})$ was aspirated from the iliac bone. Mononuclear Cells (MNCs) were obtained using density gradient separation method. The MNCs were checked for CD34+ by FACS analysis and viable count was calculated and found to be about $98 \%$. Approximately $56 \times 10^{6}$ MNCs were immediately injected post separation, intrathecally in L4-L5 using a lumbar puncture needle and catheter. The patient was also given rehabilitation therapy which included occupational therapy, psychological therapy and speech therapy. Neurofacilitation may be one of the underlying mechanisms of rehabilitation to promote recovery and functional independence. During rehabilitation sessions, effective motor learning strategies with task oriented training, for real life environment were utilized and successful attainment of functional outcomes were achieved. He was evaluated at regular intervals over the period of 18 months. After the procedure, no adverse events were recorded.

\section{Results}

The patient showed some immediate improvements within a week and significant improvements over a period of 18 months. On follow up after three months, the hyperactivity had reduced by $20 \%$ and selfstimulatory behaviour of hand flapping and touching himself in private parts diminished. His eye contact had improved. The score on Indian Scale for Assessment of Autism (ISAA) reduced from 111 (Moderate autism) to 96 (Mild Autism). On six month follow up, hyperactivity had further reduced by $70 \%$ and he could maintain meaningful eye contact. Self stimulatory behaviour had subsided completely. $\mathrm{He}$ could remember routes as his memory improved. He developed efficiency in doing his daily activities of living. New task learning abilities, concept forming and problem solving had improved. This led to increased participation in household work and performing chores independently, able to perform tasks such as unlock and lock the door without assistance. The awareness of surroundings had increased. On 18 months follow up, hyperactivity had resolved and motor repetitive movements reduced drastically. All the other improvements were sustained. On repeating the PET scan, the areas which previously showed more FDG uptake, now showed less uptake. These areas were Brocas, external frontal, medial temporal pole, precentral, sensory motor, prefrontal and insula in the right hemisphere and medial prefrontal and thalamus in the left hemisphere (Figure 1). Intelligent Quotient (IQ) on The Malin's Intelligence Scale for Indian Children increased from 44 to 49.3 (Moderate Mental Retardation). The score on ISAA reduced further from 96 to 73 (Mild Autism) with improvements in social relationship and reciprocity, emotional responsiveness, speech-language and communication, behavioral patterns, sensory aspects and cognitive component (Table 1).

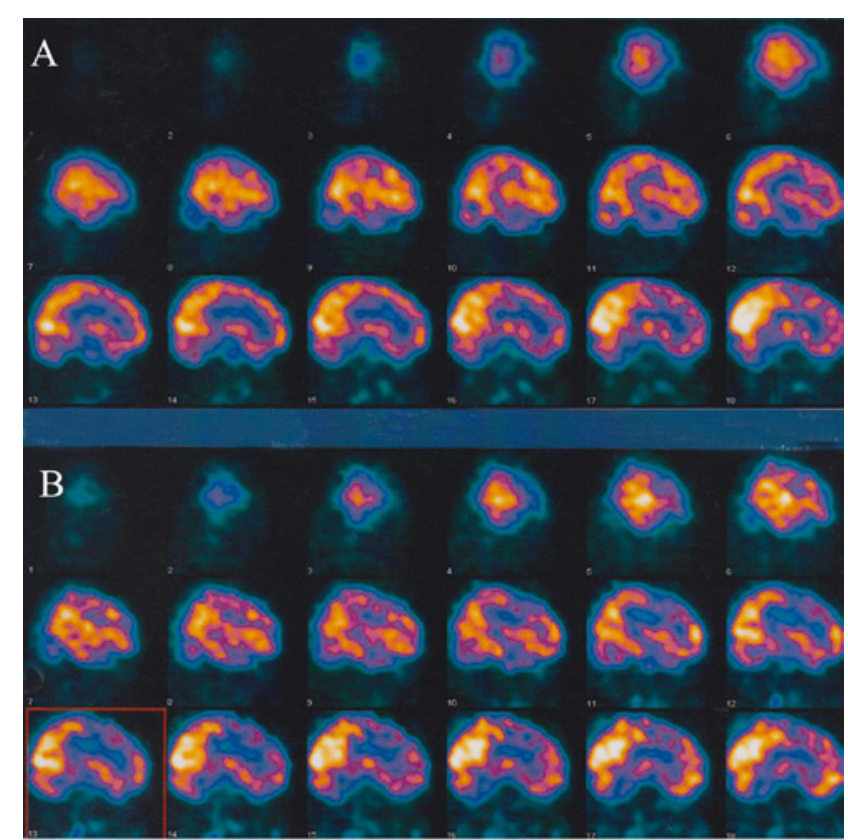

Figure 1: Comparative study of PET CT scan performed before and after cell therapy. (a) Pre cell therapy PET CT scan showing areas of hypermetabolism (more FDG uptake). (b) Post cell therapy PET CT scan showing that the areas of hypermetabolism seen in the pre-treatment scan had regressed (less FDG uptake).

\section{Discussion}

Autism spectrum disorders have many associated comorbidities such as Mental Retardation (MR); motor impairments, epilepsy, sleep dysfunction, etc. Autism and MR are closely related. About $70 \%$ of autism individuals are associated with mental retardation of varying degree [5]. Autism being one of the most complicated neurodevelopmental disorders has no definite therapeutic approach [6]. Cell therapy has been widely studied and has shown to have potential as a novel treatment for various neurological disorders [7].

This is a case study of autism treated with Autologous Bone Marrow Derived Mononuclear Cell (BMMNC) transplantation, intrathecally. These cells are a combination of hematopoietic cells such as CD 34+ and non-hematopoietic cells such as Mesenchymal Stem Cells (MSCs), tissue specific progenitor cells and stromal cells [8]. They have a unique property of migrating to the damaged areas and carry out repair process [9]. They can alter the immune system and restore the altered brain organization. These cells also exhibit paracrine effects and induce angiogenesis by producing growth factors and other chemokines such as Vascular Endothelial Growth Factor (VEGF) and Fibroblast Growth Factor (FGF2) [10].

Amongst all the physiological changes associated with autism, hypoperfusion and immune dysfunction are broadly studied [11]. Hypoperfusion results in hypoxia. Reversal of hypoxia may lead to self repair and neural proliferation, which is observed in many animal models of cerebral ischemia. In cerebral ischemia animal models, bone marrow stem cells have shown to repair the ischemia-damaged neural networks and restore the lost neuronal connections [12]. Hence, stem cells may be used to stimulate angiogenesis and lead to reperfusion. Also, in autism, there are severe immune alterations and excessive production of pro-inflammatory cytokines. Stem cells secrete several biomolecules with anti-inflammatory properties through 


\begin{tabular}{|c|c|c|c|c|}
\hline & Sub-components of ISAA & $\begin{array}{l}\text { Score Pre } \\
\text { treatment }\end{array}$ & $\begin{array}{l}\text { Score at } 3 \\
\text { month } \\
\text { follow up }\end{array}$ & $\begin{array}{l}\text { Score at } 18 \\
\text { month } \\
\text { follow up }\end{array}$ \\
\hline A. & $\begin{array}{l}\text { Social Relationship and Reci- } \\
\text { procity }\end{array}$ & 41 & 36 & 29 \\
\hline 1 & Eye contact & 5 & 4 & 3 \\
\hline 2 & Remains aloof & 5 & 5 & 4 \\
\hline 3 & Does not reach out to others & 5 & 2 & 4 \\
\hline 4 & Unable to relate to people & 5 & 5 & 4 \\
\hline 5 & $\begin{array}{l}\text { Unable to respond to social/ } \\
\text { environmental cues }\end{array}$ & 5 & 5 & 4 \\
\hline 6 & $\begin{array}{l}\text { Engages in solitary and repetitive } \\
\text { play activities }\end{array}$ & 5 & 5 & 2 \\
\hline 7 & $\begin{array}{l}\text { Unable to take turns in social } \\
\text { interaction }\end{array}$ & 5 & 4 & 2 \\
\hline 8 & $\begin{array}{l}\text { Does not maintain peer } \\
\text { relationships }\end{array}$ & 5 & 5 & 4 \\
\hline B. & Emotional Responsiveness & 13 & 8 & 5 \\
\hline 1 & Shows exaggerated emotions & 2 & 2 & 1 \\
\hline 2 & $\begin{array}{l}\text { Engaging in self stimulating } \\
\text { emotions }\end{array}$ & 5 & 2 & 2 \\
\hline 3 & $\begin{array}{l}\text { Excited or agitated for no apparent } \\
\text { reason }\end{array}$ & 4 & 2 & 1 \\
\hline c. & $\begin{array}{l}\text { Speech Language and } \\
\text { Communication }\end{array}$ & 18 & 17 & 14 \\
\hline 1 & Engages in echolalic speech & 3 & 2 & 2 \\
\hline 2 & $\begin{array}{l}\text { Produces infantile squels/unusual } \\
\text { noises }\end{array}$ & 3 & 3 & 2 \\
\hline 3 & Uses jargon or meaningless words & 3 & 3 & 1 \\
\hline D. & Behaviour Patterns & 22 & 18 & 11 \\
\hline 1 & $\begin{array}{l}\text { Shows hyperactivity and } \\
\text { restlessness }\end{array}$ & 5 & 3 & 1 \\
\hline 2 & Exhibits aggressive behavior & 4 & 3 & 1 \\
\hline 3 & Throws temper tantrums & 2 & 1 & 1 \\
\hline 4 & Insists on sameness & 5 & 5 & 1 \\
\hline E. & Cognitive Component & 7 & 7 & 4 \\
\hline 1 & $\begin{array}{l}\text { Inconsistent attention and } \\
\text { concentration }\end{array}$ & 3 & 3 & 1 \\
\hline 2 & Shows delay in responding & 2 & 2 & 1 \\
\hline
\end{tabular}

Table 1: Table giving details of the improvements in different sub components on ISAA at follow up of 3 months and 18 months.

paracrine effect. This tries to maintain equilibrium in the immune system alterations and activate endogenous repair mechanisms [13]. Thus, stem cells are capable of suppressing the pathological immune responses as well as stimulating vascularisation. Cell therapy may prove useful for the treatment of $\mathrm{T}$ cell defect associated with autism [14]. Based on these theories, Ichim et al. proposed exploration of cell therapy for autism [11]. Previous studies have shown benefits of autologous mononuclear cell transplantation in children with various incurable neurological disorders including autism. These patients reported improvement in daily functions further improving their quality of life [15].

In the present case study, on follow up, many functional improvements were recorded. These were supported by change in the ISAA scale. As there was no scale designed specific to Indian population to diagnose or measure the severity of autism, ISAA was developed by the National Institute for Mentally Handicapped (NIMH) in 2009 [16]. This scale is based on CARS and has 40 items divided under six domainssocial relationship and reciprocity; emotional responsiveness; speech, language and communication; behavior patterns; sensory aspects and cognitive component. We observed that the change in the scores on ISAA of this case study has correlated well to the clinical improvements (as described in results and Table 1).
The small increase in IQ score from 44 to 49.3 may be due to improved cognition. IQ is a higher function, so a significant change on The Malin's Intelligence Scale for Indian Children can be expected in long term and with multiple doses of stem cell transplantation. This can be explored in the future studies.

PET is a relatively non-invasive imaging test which is able to detect abnormalities in the brain based on derangements in the metabolic functions at the cellular level [17]. PET has previously shown changes with autologous BMMNCs in cerebral palsy $[18,19]$. In this case of autism, comparative PET study done at nine months after the cell therapy recorded improvements. It was observed that multiple areas of hypermetabolism (more FDG uptake) seen in the pre-treatment scan had regressed (less FDG uptake) in the scan performed after the treatment. This may suggest that the hyperactive areas of the brain were suppressed which were exhibited as clinical decrease in hyper functioning symptoms of autism. Thus, striking a balance of the overall metabolic activity of the brain. These findings are supported by the Intense World Theory, which proposes hyper-functioning of local neural microcircuits leading to hyper-perception, hyper-attention, hyper-memory and hyper-emotionality in autism [20,21].

In autism, neuronal dysfunction is evident in many regions such as Dorsolateral Prefrontal Cortex (DLPFC), the Anterior Cingulate Cortex (ACC), temporal lobe, amygdala, hippocampus, brainstem, and cerebellum [22]. Abnormalities in the Broca and Wernicke areas have been associated with impairment in social communication and language. Frontotemporal regions and amygdala have been associated with abnormalities in socioemotional processing and orbitofrontal cortex and caudate nucleus (i.e, frontostriatal system) are linked to repetitive and stereotypical behaviors [23]. Decrease in number of Purkinje cells in the cerebellar cortex has been observed in autistic brain which leads to clumsiness. The cerebellum has close anatomic connections with the frontal lobe, involving not only motor functions but also cognitive functions [24]. As seen in this case study, the PET CT scan shows improved functions of the areas discussed above. This enhanced function is seen as the clinical improvements in areas of language, social communication, behavior and cognition.

This case study of autologous bone marrow derived mononuclear cell transplantation demonstrates that this method is safe, feasible and has great potential in conjunction with current rehabilitation in treatment of autism. To our knowledge, this is one of the first case studies to have documented objective improvements in ISAA and radiological improvements in the PET study. With the pool of other studies, it will form a foundation to plan future larger trials to treat disorders such as autism.

\section{References}

1. Rubens RD, Coleman RE (1995) In: Clinical Oncology. Abeloff MD, Arnitage JO, Lichter AS, editor. New York: Churchill Livingstone. Bone metastases 643665

2. Kerin R (1983) Metastatic tumors of the hand. A review of the literature. J Bone Joint Surg Am 65: 1331-1335.

3. Flynn CJ, Danjoux C, Wong J, Christakis M, Rubenstein J, et al. (2008) Two cases of acrometastasis to the hands and review of the literature. Curr Oncol 15: $51-58$.

4. Long LS, Brickner L, Helfend L, Wong T, Kubota D (2010) Lung cance presenting as acrometastasis to the finger: a case report. Case Rep Med 2010 234289 .

5. Kontogeorgakos V, Korompilias A, Georgousis M, Papachristou D (2011) Digita metastasis presenting as infection. J Hand Microsurg 3: 25-27.

6. Kumar N, Kumar R, Bera A, Kumar P, Angurana SL, et al. (2011) Palliative and 
Citation: Sharma A, Gokulchandran N, Shetty A, Sane H, Kulkarni P, et al. (2013) Autologous Bone Marrow Mononuclear Cells may be Explored as a Novel Potential Therapeutic Option for Autism. J Clin Case Rep 3: 282. doi:10.4172/2165-7920.1000282

supportive care in acrometastasis to the hand: case series. Indian J Palliat Care 17: 241-244.

7. Chao D, Harinarayanan S, Reynolds D (2011) The acromet. J Thorac Dis 3: 209-210.

8. Borgohain B, Borgohain N, Khonglah T, Bareh J (2012) Occult renal cell carcinoma with acrometastasis and ipsilateral juxta-articular knee lesions mimicking acute inflammation. Adv Biomed Res 1: 48.

9. Rinonapoli G, Caraffa A, Antenucci R (2012) Lung cancer presenting as a metastasis to the carpal bones: a case report. J Med Case Rep 6: 384.

10. Ozcanli H, Ozdemir H, Ozenci AM, Söyüncü Y, Aydin AT (2005) [Metastatic tumors of the hand in three cases]. Acta Orthop Traumatol Turc 39: 445-448.
11. Bauer P, Saalfeld U, Schmidt G, Partecke BD (1997) [A rare case of metastasis of urinary bladder carcinoma in the pisiform bone. A case report]. Handchir Mikrochir Plast Chir 29: 154-157.

12. Heymans M, Jardon-Jeghers C, Vanwijck R (1990) Hand metastases from urothelial tumor. J Hand Surg Am 15: 509-511.

13. Ioia JV, Sumner JM, Gallagher T (1984) Presentation of malignancy by metastasis to the carpal navicular bone. Clin Orthop Relat Res : 230-233.

14. Tolo ET, Cooney WP, Wenger DE (2002) Renal cell carcinoma with metastases to the triquetrum: case report. J Hand Surg Am 27: 876-881.

15. Khokhar N, Lee JD (1983) Phalangeal metastasis: first clinical sign of bronchogenic carcinoma. South Med J 76: 927. 